\title{
"Saber de SIDA" y cuidado sexual en mujeres jóvenes de sectores populares del cordón sur de la ciudad de Buenos Aires. Apuntes para la definición de políticas de prevención
}

\author{
"Knowing about AIDS" and sexual precautions \\ among low-income women from the southern \\ area of Buenos Aires. Notes for defining \\ prevention policies
}

\footnotetext{
1 Programa de Antropología y Salud, Instituto de Ciencias Antropológicas, Facultad de Filosofía y Letras,

Universidad de Buenos Aires. CONICET Puan 470

Buenos Aires, Argentina. mgrimber@filo.uba.ar
}

\begin{abstract}
This study is part of a line of research on gender and prevention in a research program on the social construction of HIVIAIDS. We present the results of an ethnographic study among low-income women 15-35 years old in the southern area of Buenos Aires. The area has the highest number of HIVIAIDS cases and high poverty levels, extensive social degradation, and urban violence. According to our results, in the interface between "knowing about" and "behaving" there are complex processes involving stigmatized and gender-biased representations of HIVIAIDS as "other people's problem" and social and sexual relations permeated by gender stereotypes and roles. We believe that planning of prevention should be based on the consideration of overall social practices and specifically the characteristics of gender relations, prioritizing relational strategies between women and men and promoting critical reflection on the main nodes organizing daily life and active participation in the production of social relations and practices of reciprocity and equity. The increasingly precarious conditions in social life intensifies poor women's vulnerability and social interaction contexts that relate to the socioeconomic and symbolic role played by women.
\end{abstract}

Key words HIV; Acquired Immunodeficiency Syndrome; Sex Behavior; Health Policy

Resumen Este trabajo es parte de la Línea Género y Prevención de un Programa de Investigación sobre Construcción Social del VIH-SIDA. Sintetizamos en este artículo resultados del estudio etnográfico en mujeres de 15 a 35 años de sectores populares, residentes en el cordón sur de la Ciudad de Buenos Aires, área de mayor incremento de casos de VIH-SIDA, mayor pobreza, degradación y violencia urbana. Los resultados destacan que entre "saber" y "actuar" median complejos procesos que entrelazan representaciones estigmatizantes y generizadas del VIH-SIDA como "problema de otros" y modalidades de relación social y sexual, atravezadas por estereotipos y roles de género. Desde ahí sostenemos que las propuestas de prevención deben: partir del conjunto de la práctica social y, especificamente, de las relaciones de género; articular estrategias relacionales entre mujeres y varones; y promover en ellos la reflexión crítica en torno de núcleos clave de su vida cotidiana y su participación activa en la construcción de relaciones y prácticas sociales de reciprocidad y equidad. Más aún cuando la creciente precarización de la vida social intensifica la histórica vulnerabilidad de las mujeres de sectores populares, a la vez que supone crecientes contextos de interacción social que apelan y dependen del papel socioeconómico y simbólico de las mismas. Palabras clave VIH; Sindrome de Inmunodeficiencia Adquirida; Conducta Sexual; Politica de Salud 


\section{Introducción}

El crecimiento sostenido de los casos de VIHSIDA en nuestro país entre jóvenes y sobre todo entre mujeres alerta sobre las condiciones de vulnerabilidad diferencial y las tambien diferenciales posibilidades de prevención entre mujeres y varones. Como señaláramos en trabajos previos (Grimberg, 1998, 1999; Grimberg et al., 1997), el problema VIH-SIDA intersecciona núcleos estratégicos de la vida social y de las dimensiones de la subjetividad, a la vez que actualiza categorías clasificatorias morales estigmatizantes de prácticas, sujetos y grupos sociales. Pone de manifiesto, en particular, los límites de construcciones sociales que reducen la sexualidad a "reproducción" o "patología", dejando fuera, entre otros, el complejo deseo/ placer, los procesos de mercantilización, los procesos de control/subordinación en las relaciones de género.

Como han planteado distintos autores, la organización de las identidades y roles de género, las modalidades que cobran las relaciones de poder y las asimetrías sociales en el acceso y el control de recursos materiales y simbólicos entre varones y mujeres juegan un papel prioritario en relación a las posibilidades de autocuidado y a las prácticas de prevención del VIH-SIDA (Bronfman \& Minello, 1995; Campbell, 1995, 1999; Szasz, 1999). Sin embargo, la mujer y su capacidad de "negociar un sexo seguro" siguen siendo el principal foco operativo de las estrategias y programas de prevención, unilateralización que desconoce las posibilidades reales de la mayor parte de las mujeres en controlar los comportamientos de sus parejas (Campbell, 1999; Szas, 1997). Un aspecto a tener en cuenta es que las relaciones de género se articulan, a su vez, a otras desigualdades sociales tales como las de clase y étnicas, y en particular que, tanto las desigualdades en el acceso y el control de recursos materiales y simbólicos, como la autonomía y la capacidad de tomar decisiones, se hacen más problemáticos en los sectores pobres (Campbell, 1999; Farmer, 1999; Koblinsky \& Jill, 1993).

En este contexto, resulta claro que el conocimiento sobre VIH-SIDA y fundamentalmente la información técnica no guardan relación directa con las prácticas sexuales y que, a pesar de una evidente preocupación en algunas mujeres, aquéllas de sectores populares revelan límites en su capacidad de actuar de acuerdo con la información de que disponen. Las relaciones de poder y, en particular, los roles masculinos frecuentemente entran en conflicto con las orientaciones de prevención en VIH-SIDA. Ca- be preguntarse entonces por las características de los saberes y las modalidades de las prácticas, en el contexto más global de las condiciones de vida de mujeres de sectores populares.

Presentamos algunos resultados de la línea Género y Prevención de un programa más amplio de investigación sobre la Construcción Social del VIH-SIDA (líneas Procesos de Atención y Experiencia de Vivir con VIH-SIDA), desarrollado en distintas etapas desde 1994. Esta línea indaga de las relaciones e identidades de género en relación a la problemática de prevención del VIH-SIDA. Como hemos señalado en trabajos anteriores (Grimberg, 1998, 1999), entendemos el género como una construcción social e histórica de carácter relacional, que se configura a partir de las significaciones y de la simbolización cultural de las diferencias anatómicas entre varones y mujeres. Constituye una serie de asignaciones sociales que van más allá de lo biológico/reproductivo, desde las cuales se adjudican características, funciones, responsabilidades y derechos, es decir, "modos de ser" y "actuar" diferenciales (Lamas, 1986) para unos y otras, historicamente, en nuestras sociedades, produciendo y reproduciendo relaciones de desigualdad social (Grimberg, 1999). A su vez, consideramos la sexualidad desde tres dimensiones de análisis (Grimberg, 1995):

a) la consideración del complejo deseo-placer y la movilidad del deseo sexual.

b) las modalidades de intercambio sexual en relación con los modelos de familia/unidad doméstica y sus cambios, la estructuración de roles en la vida cotidiana (incluidas las modalidades de inserción laboral), los procesos de control y subordinación en las relaciones de género, etc.

c) los significados sociales asignados a la sexualidad en cada contexto histórico, en relación con los modelos de género, las modalidades culturales de expresión sexual, etc. (Standing, 1992).

\section{Notas metodológicas}

Desarrollamos un estudio etnográfico que combina entrevistas en profundidad y observación con participación con análisis de narrativas. Esta metodología prioriza: aplicar una perspectiva relacional y procesual y recuperar conceptualmente tanto la singularidad de los sujetos y los ejes de diferenciación, como los contenidos colectivos emergentes y las construcciones discursivas propias de los conjuntos estudiados; la construcción y análisis de los datos a partir de la reconstrucción de las tra- 
yectorias y del contexto global de las prácticas sociales de los sujetos de estudio, los sentidos que dan a las condiciones y modalidades de su vida, y las distintas estrategias que ponen cotidianamente en juego.

Nos centramos aqui en el estudio de mujeres jóvenes de 15 a 35 años, de sectores populares, residentes en el cordón sur de la Ciudad de Buenos Aires (barrios de San Telmo, Boca, Barracas y Lugano). Para la conformación del conjunto estudiado, utilizamos una técnica de selección por criterios que requiere la definición conceptual y operacional de las unidades de estudio mediante la identificación de categorías. Éstas fueron edad (categoría 1, de 15 a 21 años; categoría 2, de 22 a 28 años; y categoría 3, de 29 a 35 años) y relacionamientos de género (sin pareja, con pareja conviviente y con pareja no conviviente). Para ello, combinamos una estrategia de trabajo con informantes clave en el rol de "reclutadoras" y de trabajo con redes (también denominada técnica de "bola de nieve"), a partir del seguimiento de las redes de solidaridad extensa (no parientes: amigas, vecinas y conocidas) de las informantes seleccionadas en la primera instancia. Entrevistamos a un total de 35 mujeres en dos o más sesiones, con una duración variable de un máximo de 2 horas cada una; dependiendo de la disposición y de la riqueza verbal de las informantes, completamos entrevistas de entre 6 a 8 horas de duración. La línea Género y Prevención cubre, además, otra área residencial (Partido de Avellaneda, en la provincia de Buenos Aires), y, en ambas, el estudio entre varones; en total, completamos entrevistas a 67 mujeres y 50 varones en las dos zonas residenciales.

Señalamos muy sinteticamente algunas características que consideramos relevantes del conjunto estudiado. Nuestras informantes viven en inquilinatos, casa "ocupadas", piezas de hoteles y, en menor medida, en departamentos económicos alquilados. En un contexto de notable heterogeneidad en las modalidades de unidades domésticas, menos de la mitad corresponden a unidades "nucleares" - de pareja con hijos -, más de un cuarto son unidades a cargo de una mujer y, en su mayor parte, son unidades extensas. Proceden de la Provincia de Buenos Aires, de otras provincias y de países limítrofes.

Salvo las menores de 20 años, la mayor parte se encontraban conviviendo en pareja con más de 5 años de relación; las mujeres casadas representaron una notable minoría; según relataron, el casamiento es un punto de llegada, una decisión posterior, tras períodos relativamente largos de convivencia e hijos. Los hijos son una característica común (dos tercios tienen hijos); en un número significativo de casos, entre seis y ocho hijos. Más de dos tercios han tenido su primer hijo en edades tempranas: siete entre los 14 y 17 años, y once entre los 18 y los 20 años, más de la mitad "solas" (sin compañero), ya sea con o sin apoyo familiar.

En su mayoría tienen estudios secundarios incompletos (diez y seis), un cuarto completó este nivel, tiene estudios terciarios sin completar o estaban realizándolos en el momento de nuestro trabajo de campo (nueve). Es significativo el número de informantes que no supera la escolaridad primaria (completa, siete casos e incompleta, dos casos) si se tiene en cuenta que se trata de población urbana y joven. Aproximadamente la mitad tenía algún tipo de ocupación remunerada, casi un cuarto expresó estar "desocupada" y las restantes manifestaron ser "amas de casa" o "estudiar" en el momento del trabajo de campo. En su mayor parte son asalariadas en modalidades precarias e inestables de condición laboral (empleos temporarios en empresas de limpieza de oficinas, empleadas domésticas, tareas de limpieza en instituciones públicas o en programas sociales, etc).

\section{Saber de SIDA}

Si bien, en términos generales, el conjunto estudiado pone de manifiesto información sobre el VIH-SIDA y sus formas de trasmisión, el peso de categorías normativo-morales fuertemente estigmatizantes de sujetos y comportamientos introduce confusiones y notables ambivalencias en el contexto de las representaciones y de las prácticas. Con el objetivo de permitir una mirada de conjunto, puntualizamos de manera sintética tres dimensiones de este "saber": las definiciones, las formas de trasmisión y las formas de prevención.

\section{Las definiciones}

El SIDA emergió espontáneamente como problema durante distintos tramos de las entrevistas, en particular en los referidos a "las enfermedades más graves desde el punto de vista social" y "los problemas más importantes del barrio". Desde el primero, "enfermedades más graves desde el punto de vista social", el VIH-SIDA encabezó, a notable distancia de otras como "cáncer", "meningitis", "tuberculosis", "alcohol" y "droga”. A su vez, en el tramo de análisis de los "problemas mas importantes del barrio", el VIH-SIDA resultó colocado detrás de problemas como trabajo/empleo y vivienda, siguien- 
do a la mención de inseguridad, violencia, drogas o alcohol. Este posicionamiento se mantiene cuando se consideran los "problemas de salud del barrio", detrás de la mención de problemas como "droga" y "alcohol".

El conjunto de estas referencias ponen de manifiesto una percepción simultánea del VIH-SIDA: como enfermedad y como problema de seguridad. Significativamente, apareció muy debilmente en aquellos tramos autoreferenciales, por ejemplo, relatos de relacionamientos y relaciones sexuales, problemas que le preocupaban personalmente, temas hablados con la pareja o los amigos, etc. En este marco, si bien su delimitación como problema social resulta clara, se ubica más como un problema de "otros", externo al espacio particular de sus involucraciones.

La mayor parte de nuestras informantes utilizó la denominación de "el SIDA" para referirse al problema, utilizando términos como: "enfermedad infecciosa/contagiosa; no hay curales mortal; los medicamentos no curan, prolongan la vida/mejoran la vida/impiden que se enfermen y se mueran; venérealenfermedad de trasmisión sexual; es un virus; consumeladelgaza/mancha la piel/palidez/se cae el pelo/fiebre/desgano/decaimiento/ganglios".

La clave de la representación está en su configuración como enfermedad infecciosa. En este carácter, es identificada como enfermedad de trasmisión sexual, ya sea bajo esta denominación o la de "venérea".

"para mí son todas enfermedades venéreas; el Sida también lo considero como una enfermedad venérea aunque no es totalmente así, porque las otras se curan" (S., 34 años, pareja conviviente).

\section{Las formas de trasmisión/contagio}

Un aspecto a destacar es que, si bien los términos "trasmisión" y "contagio" fueron alternativamente utilizados durante las entrevistas, el peso se concentró en este último.

"el SIDA se contagia por la droga, aquí todos estos chicos que se estan drogándo hacen cualquier cosa, también se emborrachan/qué sabés con quien se acuestan" (S., 27 años, pareja conviviente).

"mucho no sé, pero en mi cuadra, dónde yo vivo, murieron bastante personas de SIDA que se juntaban enfrente o en la esquina, que se drogaban/realmente yo no sé si tenerles lástima o bronca, porque no sé" (P.,18 años, sin pareja).

Las informantes refirieron de distintas maneras a las formas de trasmisión del VIH-SIDA. Su análisis permite distinguirse tres clases: a) Una primera refirió a vias de trasmisión: "via sexual/contacto con semen"; "vía sanguinea/contacto con sangre/por la sangre/sangre con sangre"; "de madre a hijo".

b) Una segunda restringió a prácticas específicas: "relación sexual sin preservativo"; "usar jeringas sin cambiar agujas/compartir jeringas"; "hacer transfusiones con sangre no esterilizada"; "usar elementos quirúrgicos no esterilizados".

c) Una tercera, por último, remitió a prácticas generales: "relación homosexual"; "relación anal"; "penetración"; "jeringas/inyecciones"; "inyectarse/picarse"; "drogarse".

\section{Las formas de prevención}

En esta tercera dimensión del "saber", se evidencia un reconocimiento claro de medidas específicas de prevención, tales como: "usar preservativos", "no compartir jeringas/cambiar agujas", "usar material descartable", etc.

"cómo se previene para míly cómo se previene, o sea, tenés dos opciones o no te acostás con nadie (risas), este, o te ponés profilácticos o si trabajás en un lugar público de atención médica obviamente tomando todas las precauciones, o sea, no tocar/sabiendo que hay un infectado de SIDA, o sea, una aguja, no la vas a ir a tocar así" (A., 24 años, pareja no conviviente).

"no sé si es una regla segura, pero, bueno, tomando ciertas precauciones (...) no tener relaciones sexuales sin protección, ... cerciorarse que jeringas y esas cosas sean descartables y, bueno, hasta donde yo sé, tomando ese tipo de precauciones no sé si habrá otras formas de con... yo creo que que son ésas, pero, bueno, no sé si habrá otras" (M., 25 años, pareja conviviente).

Sin embargo, al mismo tiempo se ponen de manifiesto visiones "preventivas", de un elevado nivel de generalidad y fuerte contenido prescriptivo de orden moral: "cuidarse"; "hacer lo debido"; "saber con quién estás"; "no drogarse".

Sobre todo en aquéllas relacionadas globalmente con la sexualidad o con las modalidades específicas de relacionamientos de género: " $e l$ SIDA, cómo se previene y, bueno, es/no drogarse lo más importante, no y.../y no hacer cosas malas, que sé yo, no tener relaciones con otros/sólo con su pareja" (S., 27 años, pareja conviviente).

Quizás lo más significativo aquí es destacar la definida gravitación de una construcción que asocia "pareja estable"/"conocimiento-confianza" con "seguridad de no contraer" el VIH.

"Porque, bueno, ahoralya no tanto porque los hombres capaz que tienen más la precaución, antes la gente no era de tener una pareja constante no y...ly si los hombres, no todos los 
hombres, pero la mayoría, son de cambiar de pareja/ahora por el sida se cuidan más, sil hay que evitar las parejas ocasionales/creo que más no/cuando tenés tu pareja permanente, le tenés confianza" (L., 28 años, sin pareja).

"cuidándote y porque, si hay gente que tenga SIDA, es porque van y están con otras mujeres o hombres, no con las parejas estables, para mí es eso" (V., 20 años, pareja conviviente).

En síntesis, resulta claro la presencia de representaciones que combinan componentes de carácter cognitivo, por ejemplo, la identificación de formas de trasmisión definidas por el discurso biomédico, con otros de carácter prescriptivo-moral, a partir de la asociación de la enfermedad a prácticas socialmente sancionadas como "promiscuas" o "transgresoras" en referencia a la sexualidad y al uso de drogas. El peso de categorías normativo-morales fuertemente estigmatizantes de sujetos y comportamientos refuerza visiones generalizantes que globalizan sin distinguir prácticas específicas de trasmisión ("descontrol", "promiscuidad", "drogarse", "relaciones sexuales ocasionales", "relaciones sexuales con desconocidos") y de prevención ("cuidarse", "no drogarse", "pareja estable", "fidelidad", etc.).

\section{Las prácticas: estereotipos y roles de género}

En esta investigación, nos aproximamos a la sexualidad (Grimberg, 1999) como proceso que no sólo se desenvuelve, sino se construye en una historia con otros. Para esta presentación, sintetizamos algunos de los hitos más significativos, de manera a mostrar el peso de las construcciones genéricas en términos de estereotipos y roles en las relaciones sexuales.

a) La mayor parte de nuestras informantes han tenido su iniciación sexual aproximadamente entre los 15 y los 17 años, en ocho de ellas más temprano (un caso de 12, otro de 13 y seis de 14 años). En todos los casos, por primera relación sexual las entrevistadas entienden penetración vaginal.

En particular en aquéllas mayores de 20 años, su sexualidad se encuentra fuertemente asociada a la vida reproductiva. De éstas, diez manifestaron haber quedado embarazadas de su "primer novio" y ocho de parejas posteriores sin contar la actual. La categoría 2 , de mujeres entre 22 y 28 años, resultó particularmente significativa, ya que seis habían tenido su primer hijo solas, con o sin apoyo familiar, mediando o no un abandono directo por parte del varón. Otra, embarazada de su "primer novio" a los 15 años, inició la convivencia a partir de ese momento, mientras que una octava se encontraba embarazada y "sin pareja", conviviendo con sus padres, en el momento del trabajo de campo. A su vez, ocho de ellas (cuatro de la categoría 2, cuatro de la categoría 3) relataron haber sido "abandonadas" embarazadas, ya sea por su "primer novio" o por una pareja posterior.

b) En su mayoría mantienen relaciones sexuales con cierta periodicidad (sólo tres de las más jóvenes, menores de 19 años, no habían completado su iniciación sexual en el momento del trabajo de campo). En torno de las mismas confluyen distintas denominaciones, entre las que se destacan: "hacer el amor"; "relación sexual"; "coger"; "transa completa"; "hacer/tener sexo"; "ir a la cama"; "acostarse"; "encamarse"; "curtir".

Salvo para "transa completa" o "curtir", termininología utilizada por las más jóvenes, las denominaciones espontáneas no muestran diferencias por edad.

c) Las informantes remitieron a distintas modalidades de relacionamientos con términos como: "pareja", "salir con", "relaciones", "transas". Para su diferenciación, la mayoría utilizó como criterio el "amor" o el "estar enamorada". Sin embargo, mientras las mayores de 25 años, sobre todo quienes estaban en pareja, hicieron más énfasis en sus relaciones de "pareja estable" y en "el amor": "con mi primer novio tuve mis dos primeras hijas/la relación duró de los 15 a los 20 años, pero nunca vivimos, él reconoció a las nenas pero no se ocupó de ellas/mi papá se enojó mucho, en cada embarazo me pegó, pero luego se encargó de las nietas/a la primera que vivió con él la quiso un montón hasta un punto que después, cuando yo me venía a Buenos Aires, él no me dejaba que la traiga porque me decia no que ella es mía y yo la crié y como se crió al lado de éllél se hacia dueño de ella/tuve otros novios: D., con el que sali 5 meses, L. com quien estuve 4 años, y ahora vivo con J." (M., 30 años, pareja conviviente).

Las menores de 25 años refirieron un mayor número de relacionamientos y encuentros sexuales en los que primaban sólo "las ganas", "el estar bien”, entre otras expresiones.

"para la mujer no es sólo sacarse la calentura (...) otra cosa es que salgas con el pibe y llegues/llegues a hacerlo/pero no siempre estás perdidamente enamorada porqueleh a veces curtis/curtir es verse más seguido, te gusta alguien y lo ves o te vas con él seguido/bueno te cuento/con dos/el año pasado y éste" (A., 17 años, sin pareja).

“además de dos parejas/yo salí con dos, porque me gustaban, tenía ganas/pero amor no 
era/les tenía confianza, eran compañeros, no me iban perjudicar" (L., 21 años, sin pareja).

Además del "amor" o "estar enamorada", "la seguridad", "la confianza” y "el conocimiento" resultan relevantes para distinguir con quién "se tiene o no" relaciones sexuales. Tal como lo expresan los testimonios presentados, predomina una visión dicotómica que distingue entre "acto sexual" o "hecho físico", encarnado en la penetración - primera unidad de sentido -, y la posibilidad de - "puede o no" incluir "amor" o "sentimientos" - segunda unidad de sentido: “la relación sexual, ... digamos, te sacás las ganas pero, no (...) más bien eh una calenturalel amor pasa por un sentimiento" (M., 25 años, pareja conviviente).

"una relación sexual se puede tener con cualquiera, que, o con alguien que te guste aunque no lo quieras, aunque que se yo, porque tenés ganas y listo. En cambio, cuando hacés el amor, es otra cosa, estás enamorada" (L. 21 años, sin pareja).

En todos los casos, una manifiesta distinción y tensión entre el orden de "lo físico" y el del "amor" define y separa "tipos" de relacionamientos. Esta contradictoriedad da cuenta de un escenario ambigüo entre lo "permitido" y lo "no permitido", bajo los términos de "normal" y "anormal": "si usted está con su pareja y la quiere a su pareja, es hacer el amor y hacer las cosas/sexualmente/yo pienso así no/porque yo estoy con mi pareja y lo quierolentonces se hacerle sexualmente e/(risas) cómo se dice, yo voy a hacer el amor con él porque yo lo quiero, es normal" (M., 17 años, pareja conviviente).

Como hemos señalado en otro trabajo (Grimberg, 1995), el análisis de las prácticas sexuales pone al descubierto el peso de una construcción biológico-moral dominante de la sexualidad - como heterosexualidad exclusivamente reproductiva - que delimita la frontera entre sexualidad "normal" y "anormal" e instituye prácticas como "naturales” y "no naturales". Esta construcción:

- sostiene una representación dicotómica de las relaciones sexuales que separa y pone en tensión "hecho/acto físico" y "afecto/amor";

- escinde y coloca el deseo, el placer y la iniciativa sexual en el varón, y la aceptación-concesión en la mujer, constituyéndose "el amor" entre éstas en requisito, garantía y justificación de la práctica sexual. Su articulación a una noción romántico-altruista del amor, coloca a la mujer en el lugar de la entrega, el sacrificio y la concesión.

"si una está enganchada y cuando póngale una vez hace el amor y después yalya piensa que todo es del hombre esselya (...) se entrega" (M., 17 años, pareja conviviente). "una amiga que es casada me dijo... lo que pasa que hacer el amor no es algo físico, tenés que entregar el alma, me dijo (risa) y eso me re quedo y no sé.../me acuerdo que me re ayudó porque no era algo físico, ni sentí que era algo acá me di cuenta y me ayudó a abrirme ¿no?" (M., 25 años, pareja no conviviente).

En este contexto, las relaciones sexuales son "necesarias" y un "complemento" del "amor": "y que la pareja (...) se quieralel sexo debe ser un complemento/que sé yo/no que sea un acto sexual puro/pero que sea algo más/no sé tampoco platónico puro amor" (T., 26 años, sin pareja).

d) Sin diferencias por edad o por modalidad de relacionamiento, y tanto desde las expectativas (modalidad esperada) como desde lo efectivamente realizado, la iniciativa y el control de la relación está en manos del varón. Esta iniciativa cubre tanto el promover (inicio), como el desenvolvimiento de la relación en términos de propuestas, posiciones, formas de prácticas, etc.

"y el hombre es el que más provoca que pase algo. Él es el que empieza. Y siempre con un juego de manos está provocando. O empieza tocando el pelo. O me agarra de atrás de la cintura" (M., 25 años, pareja no conviviente).

"Supuestamente, tiene que empezar el hombre" (N., 27 años, pareja no conviviente).

Las posiciones sexuales ocupan un lugar relevante en relación a la iniciativa y al control de la relación. En efecto, "arriba” y "abajo" no sólo son parámetros espaciales, su significación remite a: capacidad de movimiento, a márgenes de maniobras. "Arriba” y "abajo" son lugares de poder.

- Arriba = el lugar de las riendas/manija (dominación, actividad, propuestas).

- Abajo = el lugar del me hacen/piden, etc.(subordinación, pasividad, concesión).

En la mayor parte de los relatos registrados, estos lugares no son discutidos, por lo contrario, resultan naturalizados desde los estereotipos de género, formando parte de las preferencias: "la normal hacemos (él arriba)/para mi que es como que me entrego mejor/lo siento más.../se supone que es mejor para el hombre y para la mujer/yo tengo que estar muy tranquila porque me cuesta" (S., 29 años, pareja conviviente).

Articulado a este estereotipo de género, el rol de la mujer no puede, sin embargo, entenderse como pasivo. Los datos muestran una diversidad de prácticas de distinta significación, por un lado, es parte del rol de la mujer el responder de alguna manera, el "decir sí o no" o el "poner límites": "empieza él/que se yo, como a cargocearme, que empieza que me toca el pelo, que me toca la oreja, y, bueno, así me empieza a cargocear, digamos, son cosas que él sabe como 
empezar y yo le respondo" (V., 20 años, pareja no conviviente).

Por el otro, se reconoce de manera manifiesta y se hace énfasis en un rol de género explicitamente inductivo: "provocar", "seducir", "emitir señales". "después que llega de trabajar y se baña (...) lo provoco y/y él viene me abraza... yo lo miro y le digo ah dejame de joder y él viene y está y me agarra y bueno ya después...” (M.,17 años, pareja conviviente).

"Y, por ejemplo, yo este: yo duermo con/con pantalón cortito y a él le encanta esolesa es la señal/cuando no quiero: ponele, no le doy bolilla, me pongo un pantalón largo (risas) entonces él ya se da cuentalo me pongo un jogging o cualquier cosalo sino me pongo un pantaloncito cortito, si a él le gusta" (S., 27 años, pareja conviviente).

El peso de un "deber ser como mujer", por ejemplo, el expresar desconocimiento e inexperiencia sexual, "el tener que esperar", "hacerse la difícil", "poner resistencia", etc., instituidos como "comportamientos esperados" y necesarios para el desempeño de los roles masculinos, limitan la iniciativa y la capacidad de negociación de las mujeres, ya sea como componentes del imaginario o como experiencias concretamente vividas: "si pero vos tenés que dar en ese momento/liberate dice (...) pero no puedo... que se yo/son tantas las cosas que a uno...lyo pienso siemprelqué pensará él, porque siempre te dicen capaz que vos lo hiciste con el otro/oh yo le digo, así mejor me quedo piola/viste y no es así capaz que yo quiero hacer algo nuevo o/no sé empezar/una vuelta estábamos/ teníamos una relación y ya íbamos a empezar y yo empecé, yo empecé ese día no, y que se yo, y me dice asi seguro que así habrás hecho con el otrolah, cuando me dijo así, yo dije nunca más empiezo nada/son cosas que te trauman no/porque, que se yo/porque tuviste una pareja anterior, él piensa que capaz que con la otra pareja, capaz lo que con el otro si conmigo no" (L., 28 años, sin pareja, separada).

e) Las relaciones sexuales constituyen simultáneamente algo no siempre deseado por nuestras entrevistadas. Estos estereotipos dificultan reconocer el propio deseo, a la vez que legitiman prácticas coercitivas por parte del varón como parte "natural" del rol de la mujer. En efecto, nuestros datos muestran a la iniciación sexual como un proceso fuertemente contradictorio, al que "se llega" sobre todo por iniciativa o presión del varón, y a la que se suma, en algunos casos, las presiones de pares, amigas, primas, etc., "que ya habían debutado".

"yo no quería primero, pero él insistía./Tenía miedo, vergüenza, y miedo/no sé, al dolor" (C., 18 años, pareja conviviente). “y bueno (...) que me daba miedo, tenía vergüenza, todo junto. Por eso no, no me decidía. $O$ sea, yo ya le había dicho que sí, pero no sé por qué no, no entraba al hotel, yo. Tambien tenía una presión de que tenía que hacer algo, tenía dos amigas que ya habían debutado y hablábamos/ellas me decían tambien que no tuviera miedo" (M., 25 años, pareja conviviente).

Los indicadores de coerción abarcan una amplia gama de situaciones, desde la aceptación de la relación en sí como "obligatoria" (una clara referencia se encuentra en el siguiente primer testimonio), pasando por acceder a su inicio frente a algún tipo de presión, o a posiciones o modalidades de prácticas no deseadas durante su desarrollo.

"si digo que no quiero tener relaciones, mi esposo comienza con los celos/y dice que ando con otro hombre" (G., 26 años, pareja conviviente).

"pensaba que no iba a tener más por el momento/no iba a tener más hijos, estaba muy decidida, pero despues no me dio tiempo ni de decidirme, despues que tuvo una [primera relación] se terminaron las decisiones mias (...) no sé si los desea, pero es que no se controla y por eso discutimos también/porque yo le digo quelque todo tiene un límite este, no es cuestión de todo sexual, no es/(...) a veces hay que complacerlo para no aguantarlo/que sé yo/de mal humor, porque lo noto raro" (S., 29 años, pareja conviviente).

Parte significativa de las informantes narró situaciones de tensión y distinto grado de conflictividad, asi como distintas maneras de responder frente a la presión: "no, no, yo, cuando él pide, no le hago/yo me tengo que sentir a gusto [que hace él en ese caso?] me cargosea, me sigue pidiendo/siempre vuelve a pedir (...) [y vos entonces que hacés] y según, a veces si yo (...) le hago/pero no/una pasadita nada más y ya estálpara que se deje de joder" (N., 35 años, pareja conviviente).

"si le llego a decir que no/insiste un poco, pero si sigo con el que no, él se juega a que yo ceda (...) y bueno/a veces/sé que a él es algo que le gusta practicar, entonces a veces digo que si para que él esté bien; si él está bien, yo estoy bien" (S., 34 años, pareja conviviente).

f) Como hemos señalado en un trabajo anterior (Grimberg,1999), si bien el conjunto de las entrevistadas evidencia disponer de importantes elementos cognitivos en relación al cuidado sexual, y al preservativo como forma de protección ("del embarazo", de "enfermedades"/ "infecciones sexuales" y del "SIDA", en ese orden), su utilización resulta sumamente escasa, tanto en la iniciación como en los posteriores relacionamientos sexuales. 
El uso de preservativo es una práctica eventual. En primer término, esta acotada a los momentos iniciales de una relación de pareja o a relacionamientos ocasionales; en segundo, se utiliza de forma alternada con otros métodos de cuidado o simplemente con ninguno, en los casos de relaciones definidas como "estables".

Entre otros, el relato de las informantes destaca:

- Una representación del preservativo como masculino, un "asunto de hombres", iniciativa y responsabilidad del varón (uso efectivo, compra, tenencia, momento y características de su colocación), a partir de la cual la capacidad de intervención de la mujer se reduce a solicitar.

- Una aceptación naturalizada de la resistencia del varón y/o su directa negativa al uso, a partir de una identificación negativa en términos de placer, sobre todo con énfasis en el varón ("él no siente").

- La relación uso = encuentros ocasionales o relaciones con "desconocidos"/no uso = "conocimiento", "confianza", "pareja estable" revela una línea de significación en la que "conocimiento" y "confianza" no sólo refieren a un saber "de enfermedades", sino, sobre todo, a un saber de la "conducta" del otro. Un saber moral, cuyos soportes básicos son las nociones de estabilidad y fidelidad.

\section{"Saber de SIDA" y prevención: consideraciones para la definición de estrategias y programas}

Los resultados alcanzados muestran que, entre la percepción y la categorización del problema VIH-SIDA y las prácticas, median modos de vida, así como modalidades específicas de relación social y sexual en las que son relevantes los estereotipos y roles de género, particularmente entre las mujeres de los sectores más precarizados. En efecto, en el marco de las relaciones de poder que organizan las relaciones de género, la fuerza de un "deber ser como mujer", instituida en "comportamientos esperados" y "necesarios" para el desempeño de los roles masculinos, limita la iniciativa y la capacidad de negociación general de las mujeres y, en particular, las posibilidades de negociar sexo seguro.

Estos estereotipos dificultan reconocer el propio deseo, a la vez que legitiman prácticas coercitivas por parte del varón como aspecto "natural" del rol de la mujer. La articulación a otras desigualdades sociales en relación con el acceso y el control de recursos materiales y simbólicos, o la autonomía y la capacidad de tomar decisiones, constituyen condiciones de vulnerabilidad específica en las mujeres jóvenes de sectores populares.

Al mismo tiempo, debe reconocerse que la centralidad de la noción de "contagio" y el contexto normativo-moral que asocia VIH-SIDA a "droga" /"promiscuidad" /"homosexualidad" sostienen la percepción del problema como "problema de otros", refuerzan el imaginario de garantías y seguridades morales, y diluyen las posibilidades de prevención.

Las actuales orientaciones de prevención, si bien han avanzado en la crítica a modelos pedagógico-prescriptivos, no presentan dispositivos de contención del contexto general de las condiciones de vida y de relación de las mujeres, no reconocen operativamente la complejidad de las prácticas sexuales y de los mecanismos de control que las mismas involucran o ignoran en la práctica, los modelos culturales sobre la sexualidad y las identidades sexuales, así como los aspectos vinculados al complejo deseo-placer en la mujer.

Mirada de conjunto, la problemática del VIH-SIDA en relación con la mujer descubre la gravitación de procesos socioculturales y políticos que naturalizan la sexualidad, la pareja, la maternidad, etc, oscurecen los aspectos de subordinación de las relaciones de género y, sobre todo, tienden un velo que no permite ver (y apropiar) las resistencias y las pequeñas iniciativas o transformaciones que las mujeres realizan cotidianamente.

Desde ahí sostenemos que las propuestas de prevención deben partir del conjunto de la práctica social y, sobre todo, de la especificidad de las relaciones de género; articular estrategias relacionales entre mujeres y varones; $\mathrm{y}$ promover en ellos la reflexión crítica en torno de núcleos clave de su vida cotidiana y su participación activa en la construcción de relaciones y prácticas sociales de reciprocidad y equidad. Más aún cuando la creciente precarización de la vida social intensifica las condiciones históricas de vulnerabilidad de las mujeres de sectores populares, e incrementa contextos de interacción social (desde las redes de parentesco y solidaridad a programas sociales gubernamentales y no gubernamentales) cuya actividad apela y depende del papel socioeconómico y simbólico de las mujeres.

A nuestro criterio, la prioridad, en términos de políticas de prevención, debe ser la elaboración de programas multiestratégicos - medios de comunicación, de información sistemática/contínua y actividades de discusión-reflexión personalizadas y participativas - de promoción de la salud de manera amplia, centra- 
dos en la reducción, más que en "la eliminación de los riesgos”. En tal sentido, deben partir de, y referir de manera explícita a las vivencias, a los modos de interpretación y a las necesidades de los sujetos a los que se dirigen. Para eso, deberán ocuparse de lo que los sujetos consideran cierto o válido respecto de sus propias vidas, sus preocupaciones y sus aspiraciones, así como los dilemas que enfrentan en su vida cotidiana. Ello supone elaborar y promover distintas alternativas de prevención, de acuerdo con la diversidad de los conjuntos sociales, sus vivencias y problemas cotidianos.

\section{Referencias}

BRONFMAN, M. \& MINELLO, N., 1995. Hábitos sexuales de los migrantes temporales mexicanos a los Estados Unidos. Prácticas de riesgo para la infección por VIH. In: Sida en México. Migración, Adolescencia y Género (M. Bronfman, org.), pp. 15-90, México, DF: Información Profesional Especializada.

CAMPBELL, C., 1995. Male gender roles and sexuality implications for women's Aids risk and prevention. Social Science and Medicine, 41:197-210.

CAMPBELL, C., 1999. Women, Families \& HIV-AIDS. A Sociological Perspective on the Epidemic in America. Cambridge: Cambridge University Press.

FARMER, P., 1999. Infections and Inequalities. The Modern Plagues. Berkeley: University of California Press.

GRIMBERG, M., 1995. Sexualidad y construcción social del Sida. Las representaciones médicas. Cuadernos Médico Sociales (Rosario), 70:37-51.

GRIMBERG, M., 1998. Relações entre epidemiologia e antropologia. In: Antropologia da Saúde. Traçando Identidade e Explorando Fronteiras (P. Alves \& M. Rabelo, org.), pp. 95-106, Rio de Janeiro: Editora Fiocruz.

GRIMBERG, M., 1999. Sexualidad y relaciones de género: Una aproximación a la problemática de la prevención al VIH-SIDA en sectores populares de la ciudad de Buenos Aires. Cuadernos Médicos Sociales (Rosario), 75:65-76.
GRIMBERG, M.; MARGULIES, S. \& WALLACE, S., 1997. Construcción social y hegemonía. Representaciones médicas sobre el Sida. Un abordaje antropológico. In: Sida y Sociedad (A. Kornblit, org.), pp. 107-123, Buenos Aires: Ediciones Espacios.

KOBLINSKY, J. \& JILL, G., 1993. The Health of Women: A Global Perspective. Boulder: Westview.

LAMAS, M., 1986. La antropología feminista y la categoría género. In: Nueva Antropología (L. Gortari, org.), v. 8, no 30, pp. 173-198, México, DF: Consejo Nacional de Ciencia y Tecnologia/Universidad Autónoma Metropolitana Iztapalapa.

STANDING, H., 1992. AIDS. Conceptual and metholological issues in researching sexual behavior in Sub-saharian Africa. Social Science and Medicine, 34:475-483.

SZASZ, I., 1999. Género y salud. Propuestas para el análisis de una relación compleja. In: Salud, Cambio Social y Política. Perspectivas desde América Latina (M. Bronfman \& R. Castro, org.), pp. 109121, México, DF: Instituto Nacional de Salud Pública/Foro Internacional de Ciencias Sociales y Salud/Edamex. 